\title{
Exotic Elko on string-like defects in six dimensions
}

\author{
D. M. Dantas* and C. A. S. Almeida ${ }^{\dagger}$ \\ Universidade Federal do Ceará (UFC), \\ Departamento de Física, Campus do Pici, \\ Caixa Postal 6030, 60455-760, Fortaleza, Ceará, Brazil \\ Roldão da Rocha ${ }^{\ddagger}$ \\ Centro de Matemática, Computação e Cognição, \\ Universidade Federal do ABC, 09210-580, Santo André - SP, Brazil
}

\begin{abstract}
We analyse the trapping of eigenspinors of the charge conjugation operator with dual helicity (Elko), in thin and thick string-like models with codimension-2. Elko spinor fields describe mass dimension one fermions in four dimensions (and, correspondingly, mass dimension two fermions in six dimensions), that represent natural dark matter prime candidates. This dark spinor has many applications, from particle physics to cosmology. On the other hand, six-dimensional brane-world models have, among other prominent features, the spontaneous confinement of free spin 1 fields and a mechanism that explains the mass hierarchy of fundamental fermions. In this paper, we use scalar couplings in order to confine the zero mode of Elko in six dimensions. Moreover, we use the Elko dark spinor features to propose an exotic coupling in order to remove the complex-valued terms in the massive Kaluza-Klein modes. Hence, we show that six dimensional models can resolve the main issues of Elko fields confinement presented in five dimensions.
\end{abstract}

Keywords: String-like defects, Elko spinor fields, six-dimensional braneworld models

\footnotetext{
*Electronic address: davi@fisica.ufc.br

†Electronic address: carlos@fisica.ufc.br

${ }^{\ddagger}$ Electronic address: roldao.rocha@ufabc.edu.br
} 


\section{INTRODUCTION}

Warped brane-world scenarios, like the Randall-Sundrum (RS) models [1, 2], proposed that our world is constituted by a three-brane embedded in a warped higher dimensional space-time. With this proposition, one can solve the hierarchy problem and explain several issues in various branches of Physics [1-10]. Focusing on the recent publications, brane-worlds models were used to: perform bounds into corrections to Coulomb's law $[3,4]$ and to set limits in the electrical conductivity [5], studied by the informational entropy [6-8], to respond to anomalies in the meson $B$ decay [9], and to explain issues in the neutrinos physics [10], for instance.

This paper focus on six-dimensional (6D) anti-de Sitter $\left(A d S_{6}\right)$ brane-worlds, which have some interesting features. In fact, the mass hierarchy is solved without any requirement of fine tuning between the bulk cosmological constant and the brane tension [11], with correction $\mathcal{O}\left(d^{-3}\right)$ to the Newtonian potential [11-15] that is smaller than within five-dimensional (5D) models [2]. Moreover, 6D models support the localisation of free gauge zero modes, even in the thin brane case [16-18], whereas a scalar coupling in the $A d S_{5}$ case is demanded [4]. In Lorentz violation scenarios, a massless four-dimensional (4D) graviton can be confined in 6D [19], but it is not possible in the thin 5D model [20]. Fermions fields in 6D have some prominent applications [21], and Ref. [22] suggests defects with two angular extra dimensions, where the angular momentum in the transverse space of trapped three fermion zero modes is correlated to the three generations of fundamental fermions in 4D. Additionally, Ref. [23] uses a single fermion family in 6D to explain the mass hierarchy of neutrinos.

On other hand, Elko spinor fields (dual-helicity eigenspinors of the charge conjugation operator) [24-29] are spin-1/2 matter fields, with features that put such fermionic matter fields as prime candidates to describe dark matter [30-35]. Such spinor fields have mass dimension one (in 4D), hence Elko interactions with the standard model matter and gauge fields are suppressed by at least one order of magnitude of unification scale. This means that the interactions of Elko spinor fields are merely limited to gravitons and the Higgs field. The Hawking radiation and further properties, regarding this matter field, have been explored [36]. From the phenomenological point of view, Elko can be produced by Higgs interactions [26], and realise a particle, whose symmetries are governed by the Very Special Relativity (VSR) [37]. Some attempts to detect Elko at the LHC have been moreover proposed [26], as well as promising applications in cosmology [38, 39].

The Elko spinor has been localised in 5D brane-worlds [25, 40], where has been verified that the zero mode trapping requires scalar couplings. Hence, as usual for spinors fields in 5D [41], a 
Yukawa-type interaction between Elko spinor fields and scalar fields is used in order to confine the massless mode. In Ref. [25], a scalar field with mass value was used for the thin 5D model and the scalar kink field for the thick 5D model. Moreover, Ref. [40] proposed a geometric coupling with the Ricci scalar. However, in both cases, the massive modes present complex values, which maed it impossible to compute resonances $[25,40]$. We demonstrate in this paper that the complex-valued Elko massive eigenfunctions can be removed by a covariant derivative specific coupling. In fact, regarding spin 1/2 Dirac (or Weyl) fermions and spin 3/2 Rarita-Schwinger fermions in 6D models, minimal couplings with an electromagnetic vector $U(1)$ gauge background field are performed in order to bound the zero mode [14, 21, 42, 43]. However, since Elko is a dark spinor, there is no interaction with electromagnetic gauge field, but an exotic spin structure term (such as 1-form field representing an element of the cohomology group $\left.H^{1}\left(M, \mathbb{Z}_{2}\right)\right)$ is allowed though [44-48]. These exotic spinor structures play a prominent role in the Elko models framework, which no another mass dimension 3/2 matter field can manifest. In that regard, a mass generation mechanism for the mass dimension one spinors has been derived, by coupling with a kink, in the context of a $\lambda \phi^{4}$ field theory [44].

In the present letter, we prove that in $6 \mathrm{D}$ brane-worlds the Elko zero mode is only confined with a scalar coupling term. Moreover, in order to remove the Elko massive complex-valued eigenfunction, we introduce a 4D exotic coupling of Elko spinor fields [44, 45], which also prevents the existence of bounded massive modes. To this end, we made a brief review of string-like defects, using the Gergheta-Shaposhnikov (GS) thin model $[11,16,21]$ and the Hamilton string-cigar thick model (HC) [12-14]. Next, we show the task of the Elko spinor confinement in string-like models. Further, Elko modes issues are scrutinised in the GS model, where the calculus can be achieved analytically. We present the values of scalar fields and coupling constant in order to trap the Elko zero mode and propose a topological exotic term which allows the proper treatment of massive Kaluza-Klein (KK) modes. Hence, we point the numerical values for couplings and present the generalisation to localise the Elko spinor on any $6 \mathrm{D}$ string-like model. Finally, our main results and perspectives are summarised in the conclusions.

\section{THE ELKO MATTER FIELDS IN SIX DIMENSIONS}

The metric ansatz for $6 \mathrm{D}$ string-like models can be represented by $[11,16]$

$$
\mathrm{d} s_{6}^{2}=F(r) \eta_{\mu \nu} \mathrm{d} x^{\mu} \mathrm{d} x^{\nu}+\mathrm{d} r^{2}+H(r) \mathrm{d} \theta^{2},
$$


We consider for this ansatz the signature for the $\mathcal{M}_{4}$ metric as $\eta_{\mu \nu}=\operatorname{diag}(-1,+1,+1,+1)$. The warp factors $F$ and $H$ do solely depend upon the radial coordinate $r$, that is restricted to $r \in[0, \infty)$, whereas the angular coordinate ranges $\theta \in[0,2 \pi)$.

The Ricci scalar is given by:

$$
R=-\left[4 \frac{F^{\prime \prime}}{F}+2 \frac{F^{\prime}}{F} \frac{H^{\prime}}{H}+\left(\frac{F^{\prime}}{F}\right)^{2}+\frac{H^{\prime \prime}}{H}-\frac{1}{2}\left(\frac{H^{\prime}}{H}\right)^{2}\right] .
$$

Based on the above mentioned framework, we present the first 6D model proposed by GerghetaShaposhnikov and so-called string-like defect (GS) [11, 16, 21]. For the vacuum solution and positive string tension such model provides the following metric coefficients $[11,16,21]$ :

$$
F_{G S}(r)=\mathrm{e}^{-c r}, \quad H_{G S}(r)=R_{0}^{2} F_{G S}(r)
$$

where the parameters $c$ and $R_{0}$ are positive constants, where $c^{2}=-\frac{2}{5} \frac{\Lambda}{M_{6}^{4}}$ and $R_{0}$ is an arbitrary length scale constant $[11,16,21]$. The GS model configures a thin $A d S_{6}$ space with curvature obtained by application of Eq. (3) in Eq. (2) given by:

$$
R_{G S}=-\frac{15}{2} c^{2}
$$

In order to solve some issues involving the regularity and energy conditions, we use in this paper the regular thick model so-called Hamilton string-cigar (HC)[12-14]:

$$
F_{H C}(r)=\mathrm{e}^{-c r+\tanh (c r)}, \quad H_{H C}(r)=\left(\frac{\tanh c r}{c}\right)^{2} F_{H C}(r) .
$$

This geometry is built upon a warped product between a 3-brane and the cigar soliton space, which is a $2 \mathrm{D}$ stationary solution for the Ricci flow [12-14]. Moreover, the changes performed by this regular HC model in comparison to the non-regular GS model arise just close to the origin. Hence, asymptotically Eq. (3) is retrieved. Fig. 1 shows that the curvature of the HC model converges asymptotically to the GS curvature (4).

Now, we study the trapping of Elko fermions, where we shall conclude that there is no possibility of confining the zero mode of Elko fields in 6D models without couplings terms. Based upon the Elko 5D action of Ref. [25], the action for bulk massless Elko spinors in six dimensions is given by

$$
S=-\int \mathrm{d} x^{6} \sqrt{-g}\left[\frac{g^{M N}}{4}\left(D_{M} \bar{\Upsilon} D_{N} \Upsilon+D_{N} \bar{\Upsilon} D_{M} \Upsilon\right)+\zeta_{1} \bar{\Upsilon} \mathcal{F}_{1} \Upsilon\right]
$$

where $D_{M}$ is the covariant derivative and $\Upsilon$ represents the $8 \times 1$ mass dimension two Elko spinor field, the $\mathcal{F}_{1}(r)$ is a mass dimension two scalar field and $\zeta_{1}$ a dimensionless coupling constant. The following equation of motion is obtained:

$$
\left[\frac{1}{\sqrt{-g}} D_{M}\left(\sqrt{-g} g^{M N} D_{N}\right)-2 \zeta_{1} \mathcal{F}_{1}\right] \Upsilon=0
$$




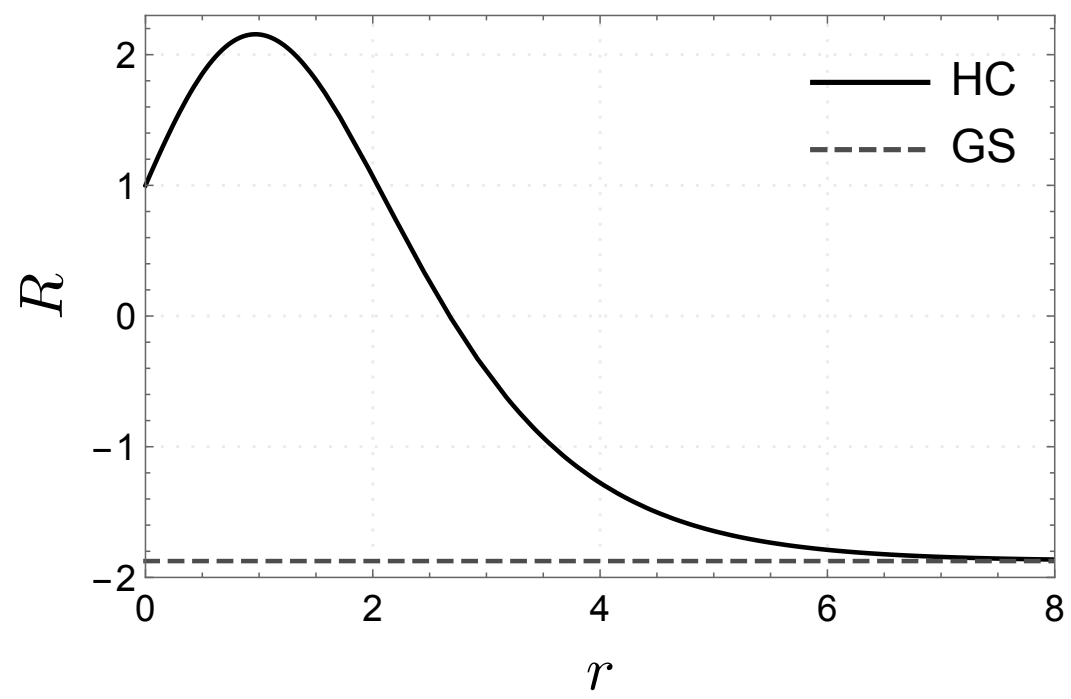

Figure 1: Ricci curvature scalar for the HC model (black line) and the GS model (gray dashed line), both for $c=0.5$.

Explicitly, in the absence of an exotic term, the covariant derivative obtained from (1) reads

$$
D_{M} \Upsilon=\left[\partial_{M}+\Omega_{M}\right] \Upsilon
$$

the non-vanishing terms of spin connections take the forms $\Omega_{\mu}(r)=\frac{1}{4} \frac{F^{\prime}}{\sqrt{F}} \Gamma_{\bar{\mu}} \Gamma_{\bar{r}}$ and $\Omega_{\theta}(r)=$ $\frac{1}{4} \frac{H^{\prime}}{\sqrt{H}} \Gamma_{\bar{\theta}} \Gamma_{\bar{r}}$, where the primes denote the derivative with respect to $r$, whereas $\Gamma_{\bar{M}}$ are the $6 \mathrm{D}$ gamma matrices in the flat space, which can be represented in terms of $4 \mathrm{D}$ flat gamma matrices $\gamma_{\bar{M}}[14,16,21]$. We choose the usual Weyl decomposition for spinors in 6D models, the 6D gamma matrices and the Kaluza-Klein spinor decomposition [14, 16, 21]

$$
\Upsilon(x, r, \theta)=\left(\begin{array}{l}
\lambda \\
0
\end{array}\right), \lambda\left(x^{\mu}, r, \theta\right)=\sum_{n, l} \lambda_{n}\left(x^{\mu}\right) \varepsilon_{n}(r) \lambda_{l}(\theta),
$$

where $\lambda_{n}(x)=\varsigma_{ \pm}^{(n)}\left(x^{\mu}\right)+\tau_{ \pm}^{(n)}(x)$ represents the four types of mass dimension one 4D Elko [25, 40]. The $\varepsilon_{n}(r)$ is the radial component which must be confined, whereas the $\lambda_{l}(\theta)=\mathrm{e}^{i l \theta}$ denotes the angular component with $l$ the orbital number $[11,16,21]$. The index $n$ labels the values of masses $m_{n}$. It is worth to mention that this spinor is indeed an eigenspinor of the $6 \mathrm{D}$ charge conjugation operator, and restricted to $4 \mathrm{D}$ is then an eigenspinor of the corresponding $4 \mathrm{D}$ charge conjugation operator. The result is interesting and it means that the KK modes of different types of an Elko spinor are the same and indistinguishable [25].

According to this decomposition, the $4 \mathrm{D}$ flat gamma matrices act upon $4 \mathrm{D}$ spinors $\lambda_{n}(x)$ as 
$[25,40]:$

$$
\begin{aligned}
\gamma^{\mu} \partial_{\mu} \varsigma_{ \pm}(x)=\mp i m \varsigma_{\mp}(x) ; & \gamma^{\mu} \partial_{\mu} \tau_{ \pm}(x)= \pm i m \tau_{\mp}(x) ; \\
\gamma^{r} \varsigma_{ \pm}(x)= \pm \tau_{\mp}(x) ; & \gamma^{r} \tau_{ \pm}(x)=\mp \varsigma_{\mp}(x) ; \\
\gamma^{\theta} \varsigma_{ \pm}(x)=\mp i \varsigma_{\mp}(x) ; & \gamma^{\theta} \tau_{ \pm}(x)= \pm i \tau_{\mp}(x) .
\end{aligned}
$$

It is worth to emphasize that Elko spinors do not obey a Dirac equation, since the Dirac spinor $\psi$ satisfies $i \gamma^{\mu} \partial_{\mu} \psi=m \psi[25,40]$.

Turning to Eq. (7) the $4 \mathrm{D}$ section yields

$$
\begin{gathered}
{\left[\frac{D_{\mu}}{\sqrt{-g}}\left(\frac{\sqrt{-g} \eta^{\mu \nu}}{F(r)} D_{\nu}\right)\right] \Upsilon=} \\
\sum_{n, l} \lambda_{n}(x) \lambda_{l}(\theta) \frac{1}{F}\left[m_{n}^{2}-\frac{i m_{n} F^{\prime}}{2 \sqrt{F}}-\frac{1}{4}\left(\frac{F^{\prime}}{\sqrt{F}}\right)^{2}\right] \varepsilon_{n}(r) .
\end{gathered}
$$

Moreover, the radial part yields

$$
\begin{gathered}
{\left[\frac{\partial_{r}\left(\sqrt{-g} \partial_{r}\right)}{\sqrt{-g}}-2 \zeta_{1} \mathcal{F}_{1}\right] \Upsilon=} \\
\sum_{n, l} \lambda_{n}(x) \lambda_{l}(\theta)\left[\partial_{r}^{2}-2\left(\frac{F^{\prime}}{F}+\frac{1}{4} \frac{H^{\prime}}{H}\right) \partial_{r}-2 \zeta_{1} \mathcal{F}_{1}\right] \varepsilon_{n}(r) .
\end{gathered}
$$

Finally, the angular component of the equation of motion is given by

$$
\begin{gathered}
{\left[\frac{D_{\theta}}{\sqrt{-g}}\left(\frac{\sqrt{-g}}{H(r)} D_{\theta}\right)\right] \Upsilon=} \\
\sum_{n, l} \lambda_{n}(x) \lambda_{l}(\theta) \frac{1}{H}\left[\frac{l}{2} \frac{H^{\prime}}{\sqrt{H}}-l^{2}-\frac{1}{16}\left(\frac{H^{\prime}}{\sqrt{H}}\right)^{2}\right] \varepsilon_{n}(r) .
\end{gathered}
$$

The sum of Eqs. (10), (11) and (12) results in a complete equation of motion (7), where the annulment condition befalls in the radial component $\varepsilon_{n}(r)$. For the $s$-wave solution $(l=0)$, we obtain the following second order differential equation:

$$
\left[\partial_{r}^{2}+\mathcal{P}(r) \partial_{r}+\mathcal{Q}(r)\right] \varepsilon_{n}(r)=0
$$

where the coefficients are:

$$
\begin{aligned}
& \mathcal{P}(r)=2 \frac{F^{\prime}}{F}+\frac{1}{2} \frac{H^{\prime}}{H}, \\
& \mathcal{Q}_{n}(r) \frac{m_{n}^{2}}{F(r)}-\frac{i m_{n} F^{\prime}}{2 F^{3 / 2}}-\left[\left(\frac{F^{\prime}}{2 F}\right)^{2}+\left(\frac{H^{\prime}}{4 H}\right)^{2}\right]-2 \zeta_{1} \mathcal{F}_{1} .
\end{aligned}
$$

To consider a solution of Eq. (13) as confined mode, it must obey certain conditions. In fact, the boundary conditions due to the axial symmetry $[11,14,16] \mathrm{read}$

$$
\varepsilon_{n}^{\prime}(0)=\varepsilon_{n}^{\prime}(\infty)=0
$$


and the orthonormality condition

$$
\int_{0}^{\infty} F(r) \sqrt{H(r)} \varepsilon_{n}^{*}(r) \varepsilon_{s}(r) \mathrm{d} r=\delta_{n s}
$$

must hold as well. Hereafter we particularise these results for the string-like brane-worlds models.

\section{CONFINING ELKO IN THE THIN MODEL}

We start to analyse the Elko matter fields on the GS thin string-like defect, where the method can be performed analytically. We expose the forms of scalar fields that provide a confined zero mode and real-evaluated massive solutions. The Eq. (13) with the warp factors of GS string in Eq. (3) yields

$$
\varepsilon_{n}^{\prime \prime}(r)-\frac{5 c}{2} \varepsilon_{n}^{\prime}(r)+\left(m_{n}^{2} \mathrm{e}^{c r}-\frac{i m_{n} c}{2} \mathrm{e}^{\frac{c r}{2}}-\frac{5 c^{2}}{16}-2 \zeta_{1} \mathcal{F}_{1}\right) \varepsilon_{n}(r)=0 .
$$

For the massless mode we have

$$
\varepsilon_{0}^{\prime \prime}(r)-\frac{5 c}{2} \varepsilon_{0}^{\prime}(r)-\left(\frac{5 c^{2}}{16}+2 \zeta_{1} \mathcal{F}_{1}\right) \varepsilon_{0}(r)=0 .
$$

In order to work with constant coefficients differential equations, we assume $\mathcal{F}_{1}$ constant, as ex-

pected for thin models [25, 40], where there is a scalar field with mass value in Ref. [25] or the constant curvature term [40]. Hence, the solution reads

$$
\varepsilon_{0}(r)=N_{1} \mathrm{e}^{\left(\frac{5 c}{4}-\sqrt{\frac{15}{8} c^{2}+2 \zeta_{1} \mathcal{F}}\right) r}+N_{2} \mathrm{e}^{\left(\frac{5 c}{4}+\sqrt{\frac{15}{8} c^{2}+2 \zeta_{1} \mathcal{F}}\right) r}
$$

where $N_{1}, N_{2}$ are real arbitrary constants with values obtained by application of conditions (16) and (17). Due to the boundary condition in Eq. (16), we have that the single value of coupling term $\zeta_{1} \mathcal{F}_{1}$ that gives a non-null solution is

$$
\zeta_{1} \mathcal{F}_{1}=-\frac{5}{32} c^{2}
$$

The physical significance of this term $\mathcal{F}_{1}$ in the thin model can be interpreted both as a squared mass value $[25,40]\left(M_{E l k o}^{2}=c^{2}\right)$ with the coupling constant $\zeta_{1}=-5 / 32$. Or else as a geometrical coupling [40] with the scalar curvature of the GS model in Eq. (4) with $\zeta_{1}=1 / 16$. In fact, the solution (20) with imposition of Eq. (21), that obeys both conditions (16) and (17), leads to a constant solution for the Elko zero mode as:

$$
\varepsilon_{0}(r)=\sqrt{\frac{3}{2 R_{0}} c}
$$


This is the same solution for the zero mode of gravity [11] and the scalar fields in 6D [14]. For massive modes, Eq. (13) in the GS string with the choice in Eq. (21) presents the following complex-valued solution:

$$
\varepsilon_{n}(r)=\mathrm{e}^{\frac{5 c}{4} r} \mathrm{e}^{-\frac{x_{n}}{2}}\left[N_{1}^{n} U\left(\frac{5}{2}, 6, X_{n}\right)+N_{2}^{n} L_{\frac{5}{2}}^{5}\left(X_{n}\right)\right]
$$

where $X_{n}(r)=4 \frac{i m_{n}}{c} e^{\frac{c r}{2}}$, the $U$ is the confluent hypergeometric function of the second kind, $L$ is the generalised Laguerre polynomial, and $N_{1}^{n}, N_{2}^{n}$ are normalisation constants. The amplitude term $\mathrm{e}^{\frac{5 c}{4} r}$ in (23) makes this expression to grow exponentially, whereas the other terms are oscillating. Hence the boundary condition (16) is not satisfied at $r \rightarrow \infty$ as can be seen in Fig. 2. Even if the boundary condition (16) is ignored, the growth of $\left|\varepsilon_{n}(r)\right|^{2}$ is slowed by $F \sqrt{H}=R_{0} \mathrm{e}^{-\frac{3}{4} c r}$ in Eq. (17). Nevertheless, this integrand still increases and can not be normalised. Thus, there is no bounded massive Elko in the GS 6D thin model. It is interesting to point out that in the 5D case, for the thin RS model, Refs. [25, 40] found Whittaker functions for Elko massive solutions, which are complex-valued as well. This complex-valued result is generated by the term $-\frac{i m_{n} c}{2} \mathrm{e}^{\frac{c r}{2}}$ in Eq.

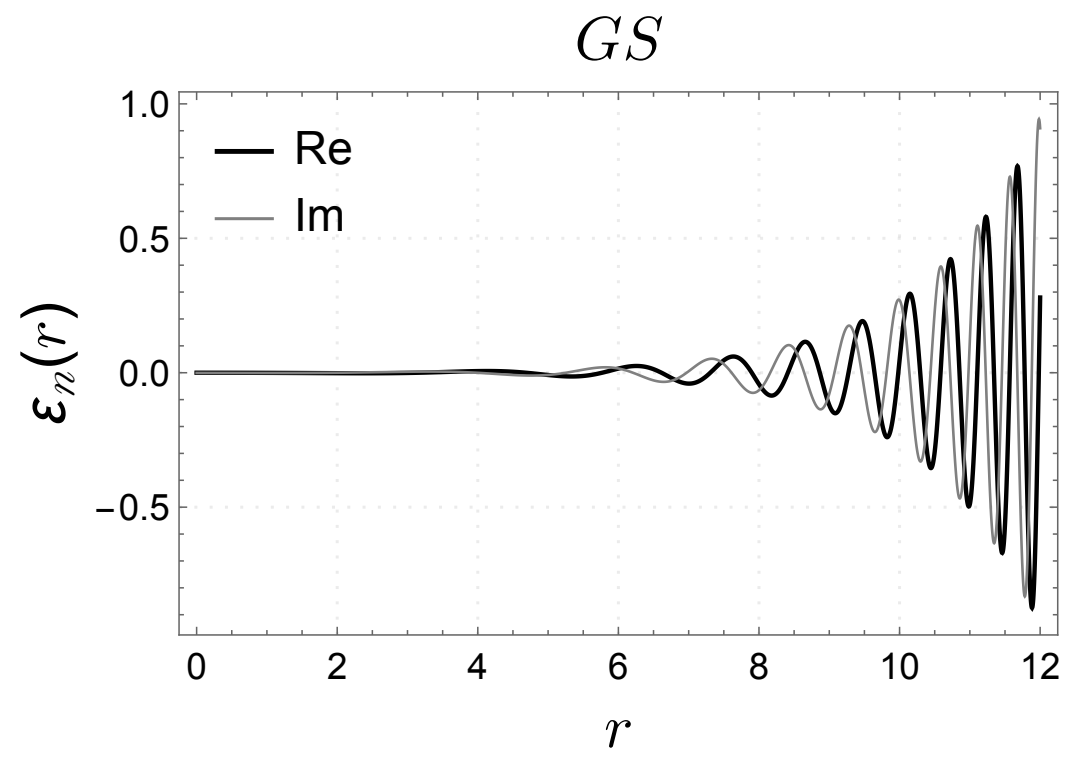

Figure 2: Solutions in real (thick line) and imaginary (thin line) parts of the eigenfunction for GS string in Eq. (23). We set $c=0.5, m_{n}=0.80, R_{0}=1.00$ and the constants $N_{1}^{n}=N_{2}^{n}=0.05$.

(18), which comes from the Elko 4D portion in Eq. (10).

A way to prevent this result is to modify the covariant derivative in similar form to proposed in Refs. [44, 45]. The covariant derivative acting on exotic spinor fields changes by an additional 1-form field that defines an integer cohomology class in the Čech sense [46-48], encrypting the non-trivial topology. The first example of a non-trivial topology is that probed by an electron in 
the vicinity of a wire with a current, which is the source of the Aharonov-Bohm effect. Considering a trivial topology is a quite strict framework [46]. In fact, the path integral setup includes multiply connected spaces $[49,50]$, also employed in the study of superconductivity, wherein exotic spin structures describe the Cooper pairing phenomenon [47]. Multiply connected spaces are employed to study Feynman propagators [51] as well as the vacuum polarization in electrodynamics [52]. Exotic spinors yield distinct effects of vacuum polarization, producing a causal photon propagation. In the context of the exotic Dirac equation, or the Dirac-like type of coupled equations that govern Elko spinors, the electromagnetic potential is affected by the transformation $A \mapsto A+\frac{1}{2 \pi i} \xi^{-1} \mathrm{~d} \xi$, namely, corresponding to a shifted electromagnetic potential, with $\xi$ being a scalar field. When mass dimension $3 / 2$ spinors (in $4 \mathrm{D}$ ) or mass dimension $5 / 2$ spinors (in $6 \mathrm{D}$ ) are regarded, the exotic term may be, hence, assimilated into any external electromagnetic potential, encoding an element of $H^{1}\left(M, \mathbb{Z}_{2}\right)$ [45, 47, 48]. Hence, an additional term in the Dirac operator induces extra degrees of freedom for fermionic particles [45]. In this case, the $4 \mathrm{D}$ portion $D_{\mu}$ in (8) can be transformed into $[44-48]$

$$
\gamma^{M} D_{M} \Upsilon\left(x^{\mu}, r, \theta\right)=\left[\gamma^{M} \partial_{M}+\gamma^{M} \Omega_{M}(r)+\zeta_{2} \mathcal{F}_{2}(r)\right] \Upsilon\left(x^{\mu}, r, \theta\right)
$$

where the exotic term $\mathcal{F}_{2}$ reads

$$
\mathcal{F}_{2}(r)=\frac{1}{2 \pi i} \xi^{-1} \mathrm{~d} \xi=\gamma^{M} \partial_{M} \Theta(r)
$$

for a scalar field $\Theta(r)[44,45]$. The exotic term in Eq. (25) originates from any non-trivial topology in the bulk $[44,45]$ and has the restriction to be normalizable. It is worth to emphasize that only mass dimension one (in 4D) and mass dimension two (in 6D) quantum fields are capable to probe exotic couplings. On the other hand, mass dimension five-halves (in 6D) quantum fields realize this term as a shift of a gauge potential $A=\gamma^{M} A_{M}$. Therefore any exotic term that might exist when gauge fields are considered in an effective Lagrangian is absorbed into a gauge potential $A^{\prime}=\gamma^{M}\left(A_{M}+\partial_{M} \Theta(r)\right)[44,45]$. Hence, the topological bulk content, provided by the second cohomology group of the $6 \mathrm{D}$ space-time to $\mathbb{Z}_{2}$, generates a physical signature, corresponding to an additional term $\partial_{M} \Theta(r)$ in the covariant derivative. Nevertheless, standard fermions perceive it as a shift on a gauge potential [45]. Consequently just mass dimension one quantum fields can indeed be affected by any exotic coupling, since they do not interact with any gauge field. Eqs. (24) and (25) comprise a gauge choice, whose just the $4 \mathrm{D}$ portion is necessary to circumvent the problems regarding imaginary values.

Similarly to proposed in Ref. [44], we can adjust the scalar fields $\xi$ in order to remove the 
complex-valued term in Eq. (18) as

$$
\xi_{G S}(r)=\exp \left[-2 \pi m \mathrm{e}^{\frac{c r}{2}}\right]
$$

which has the plot shown in Fig. 3.

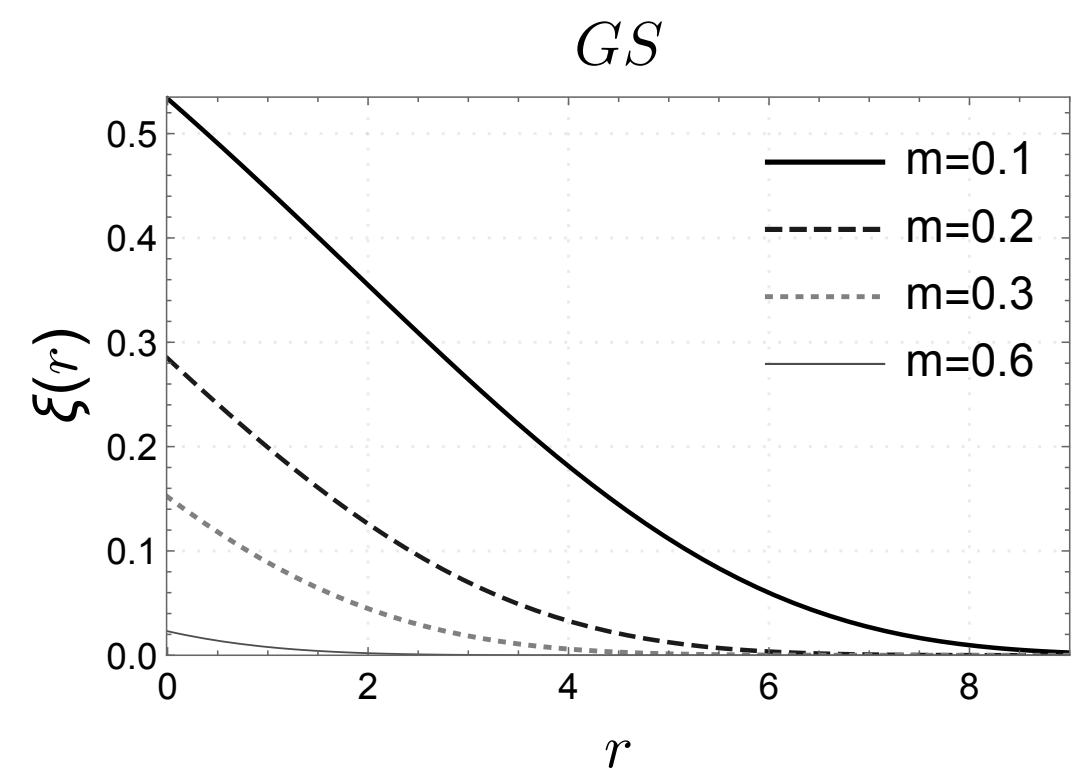

Figure 3: Scalar fields $\xi(r)$ for the GS thin model with $c=0.5$ and some mass values. All these $\xi(r)$ can be normalisable.

Indeed, the field choice in Eq. (26) applied in Eq. (25) shows an exotic coupling $\mathcal{F}_{2}(r)=$ $\frac{i m_{n} c}{2} \mathrm{e}^{\frac{c r}{2}} d r$. Since that $d r \equiv \gamma^{r}$, the term with coupling constant $\zeta_{2}=1$ cancels the imaginary term in Eq. (18). Due to it, and with the previously fixation of Eq. (21), Eq. (18) turns to be:

$$
\varepsilon_{n}^{\prime \prime}(r)-\frac{5 c}{2} \varepsilon_{n}^{\prime}(r)+m_{n}^{2} \mathrm{e}^{c r} \varepsilon_{n}(r)=0 .
$$

which has only real-valued solutions in terms of Bessel functions [8, 11-14]

$$
\varepsilon_{n}(r)=\mathrm{e}^{\frac{5 c}{4} r}\left\{N_{1}^{n} J_{\frac{5}{2}}\left(\frac{2 m_{n}}{c} \mathrm{e}^{\frac{c r}{2}}\right)+N_{2}^{n} Y_{\frac{5}{2}}\left(\frac{2 m_{n}}{c} \mathrm{e}^{\frac{c r}{2}}\right)\right\}
$$

The oscillation of these solutions (28) exponentially grows with $r$. Hence no massive mode can be trapped, as expected [8,11-14]. However, the resonance method can be applied in this case of Eq. $(28)[8,14]$, which it is not possible in the case of equation (23). Hereby, the main aim of this letter has been achieved for the thin 6D model.

In order to study the generalisation of scalar coupling $\mathcal{F}_{1}$, we can perform the conformally plane metric transformation $\mathrm{d} s_{6}{ }^{2}=F(z)\left[\eta_{\mu \nu} \mathrm{d} x^{\mu} \mathrm{d} x^{\nu}+\mathrm{d} z^{2}+\beta(z) \mathrm{d} \theta^{2}\right]$, where $\beta(z)=H(z) / F(z)$. In 
this form, changing $z(r)=\int_{0}^{r} F^{-\frac{1}{2}}\left(r^{\prime}\right) \mathrm{d} r^{\prime}$ and $\varepsilon_{n}(z)=F^{-1}(z) \beta^{-\frac{1}{4}}(z) \tilde{\varepsilon}_{n}(z)$, assuming the fixation in Eq. (26), we turn the Sturm-Liouville Eq. (13) into a Schrödinger-like equation as [8, 12-14]:

$$
-\ddot{\tilde{\varepsilon}}_{n}(z)+U(z) \tilde{\varepsilon}_{n}(z)=m_{n}^{2} \tilde{\varepsilon}_{n}(z)
$$

where the dots represent derivatives with respect to the $z$ coordinate and the analogue quantum potential $U(z)$ for the Elko has the form

$$
U(z)=\frac{\ddot{F}}{F}+\left(\frac{\dot{F}}{4 F}\right)^{2}-\frac{1}{8}\left(\frac{\dot{\beta}}{\beta}\right)^{2}+\frac{5}{8} \frac{\dot{F}}{F} \frac{\dot{\beta}}{\beta}+\frac{1}{4} \frac{\ddot{\beta}}{\beta}+2 \zeta_{1} \mathcal{F}_{1} F .
$$

On the other hand, the Ricci curvature in the conformal metric reads:

$$
R(z)=\frac{1}{F}\left[5 \frac{\ddot{F}}{F}+\frac{5}{2} \frac{\dot{F}}{F} \frac{\dot{\beta}}{\beta}+\frac{\ddot{\beta}}{\beta}-\frac{1}{2} \frac{\dot{\beta}^{2}}{\beta^{2}}\right] .
$$

Hence, we conclude in this new variable system that, unlike 5D models, a scalar coupling proportional to Ricci curvature cannot be used for a general $6 \mathrm{D}$ model. In fact, the general expression for the $\mathcal{F}_{1}$ fields, that allows the existence of Elko zero mode, must be:

$$
\zeta_{1} \mathcal{F}_{1}(z)=-\frac{1}{32 F}\left[5\left(\frac{\dot{F}}{F}\right)^{2}+\frac{\dot{\beta}}{\beta} \frac{\dot{F}}{F}+\left(\frac{\dot{\beta}}{\beta}\right)^{2}\right]
$$

which has none second derivative terms, as the present in the Ricci scalar (30). However, for the particular GS thin model this coupling can be interpreted as the curvature, due to the nonregular term $\beta(z)=$ constant, which clearly vanishes when derived. Nevertheless, in this new set of variable, the Schrödinger potential and the normalised zero mode in the GS models reads

$$
U(z)=6\left(z+\frac{2}{c}\right)^{-2}, \tilde{\varepsilon}_{0}(z)=\sqrt{\frac{24}{c^{3}}}\left(z+\frac{2}{c}\right)^{-2},
$$

allowing the confinement of massless Elko in the thin $6 \mathrm{D}$ model.

\section{ELKO IN THE THICK HC MODEL}

In the Hamilton string-cigar thick model, the factors (5) replaced into coefficients (15) of differential equation (13) read

$$
\begin{gathered}
\mathcal{P}(r)=-\frac{5 c}{2}\left(\tanh ^{2}(c r)-\frac{4}{5} \operatorname{sech}(2 c r)\right) \\
Q_{n}(r)=\frac{m_{n}^{2}}{\mathrm{e}^{-(c r+\tanh c r)}}-\frac{i m_{n} c \tanh ^{2}(c r)}{2 \mathrm{e}^{-\frac{1}{2}(c r+\tanh c r)}-2 \zeta_{1} \mathcal{F}_{1}+} \\
-\frac{5 c^{2}}{16}\left[\tanh ^{4}(c r)+\frac{4}{5}\left(2 \operatorname{sech}^{2}(2 c r)-\frac{\operatorname{sech}^{2}(c r)}{\operatorname{coth}(c r)}\right)\right] .
\end{gathered}
$$


The analytical solutions of the equation (13) with coefficients (33) are not straightforward to find, even for the massless case. However, we see that asymptotically when $r \rightarrow 0$, the coefficients (33) exhibit singularities, whereas for $r \rightarrow \infty$, the coefficients (33) converge those thin coefficients of Eq. (18), $\mathcal{P}(r \rightarrow \infty)=-\frac{5 c}{2}$ and $Q_{n}(r \rightarrow \infty)=m_{n}^{2} \mathrm{e}^{c r}-\frac{i m_{n} c}{2} \mathrm{e}^{\frac{c r}{2}}-\frac{5 c^{2}}{16}-2 \zeta_{1} \mathcal{F}_{1}$. Consequently, the massive eigenfunctions for the $\mathrm{HC}$ string are divergent at infinity, hence non-normalizable, irrespectively of the boundary condition at the origin.

For the zero mode in $\mathrm{HC}$, we can propose a generalised coupling into $r$ variable in this form:

$$
\begin{gathered}
\zeta_{1} \mathcal{F}_{1}(r)=-\frac{1}{2}\left[\left(\frac{F^{\prime}}{2 F}\right)^{2}+\left(\frac{H^{\prime}}{4 H}\right)^{2}\right], \\
=-\frac{c^{2}}{8}\left[\tanh ^{4}(c r)+\frac{1}{4}\left[4 \operatorname{csch}(2 c r)-\tanh ^{2}(c r)\right]^{2}\right],
\end{gathered}
$$

which can be put in the alternative $z$ variable form of Eq. (31) when $\frac{\mathrm{d}}{\mathrm{d} r} \mapsto F^{-\frac{1}{2}} \frac{\mathrm{d}}{\mathrm{d} z}$ and $H=\beta F$. This scalar solution of Eq. (35) is not the Ricci curvature in the Eq. (2). However, the solution in Eq. (35) has asymptomatically the same behaviour of the string-vortex scalar field of Ref. [53]. This imposition of Eq. (35) in Eq. (13) gives a generalised constant zero mode that obey the conditions (16) and $(17)$ as $\varepsilon_{0}(r)=\left[\int_{0}^{\infty} d r F(r) \sqrt{H(r)}\right]^{-1 / 2}$. This expression can be only numerically evaluated. Moreover, in order to remove the imaginary mass term, we can fix the scalar fields $\zeta_{2} \mathcal{F}_{2}(r)=\frac{1}{2 \pi i} \frac{\mathrm{d} \xi}{\xi}=\frac{i m}{2} F^{\prime}(r) F^{-\frac{3}{2}}(r) d r$ and $\xi(r)$ as:

$$
\xi(r)=\exp \left[\pi m \int_{r} \mathrm{~d} r F^{\prime} F^{-\frac{3}{2}}\right] .
$$

Fig. (4) shows that this scalar field $\xi(r)$ is regular and normalisable in the HC model, as well.

Hence, the real-valued Sturm-Liouville equation (13) turns into the HC model as:

$$
\begin{gathered}
\varepsilon_{n}^{\prime \prime}(r)-\frac{5 c}{2}\left(\tanh ^{2}(c r)-\frac{4}{5} \operatorname{sech}(2 c r)\right) \varepsilon_{n}^{\prime}(r)+ \\
+m_{n}^{2} \mathrm{e}^{(c r-\tanh c r)} \varepsilon_{n}(r)=0 .
\end{gathered}
$$

this solution was studied in Ref. [13]. The numerical value differs from the analytical solution of the thin model (28) only close to the origin [13], as can be see in Fig. 5. Working with the conformal variable $z$, the expressions for the Elko zero mode, $\varepsilon_{0}(z)$, and analogue quantum potential $U(z)$ can be only numerically-valued in the HC scenario. However, Fig. 6 shows a comparasion between the results for the GS model (32) and those for the $\mathrm{HC}$ model. In the HC model, the zero mode is also normalisable, but the $\mathrm{HC}$ potential has an infinite well at origin, as also verified in Refs. $[13,14]$. We will study the resonances in a future work. 


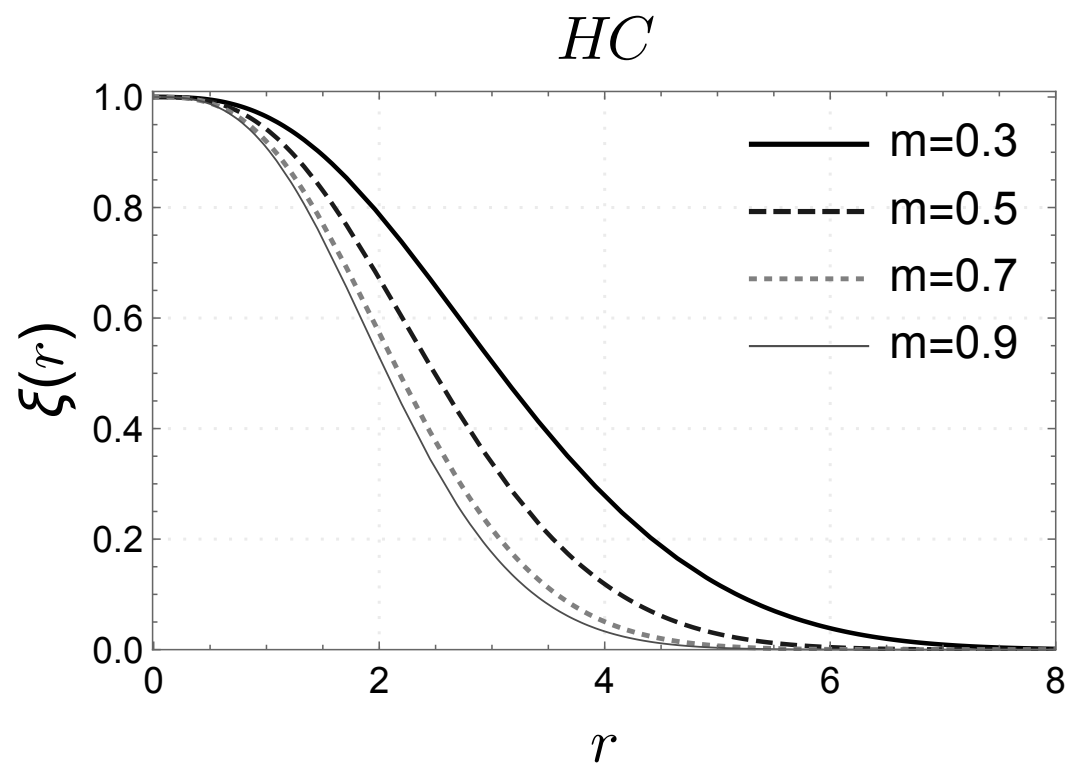

Figure 4: Numerical plot of scalar fields $\xi(r)$ for $\mathrm{HC}$ thick mode with $c=0.5$ and some mass values. All these $\xi(r)$ can be normalisable.

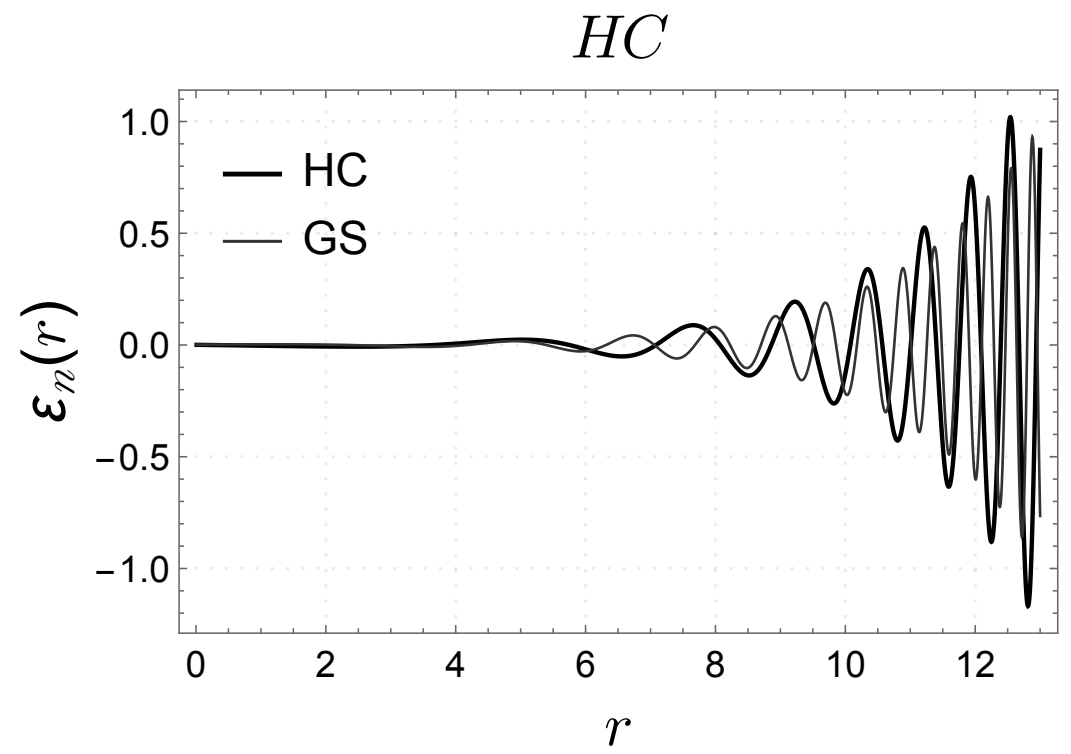

Figure 5: Numerical solution $\varepsilon_{n}(r)$ for the HC thick model (37) (black line) and for GS thin model (28) (gray line), both with $c=0.5$ and $m=0.8$. The normalisation constants are set $N_{1}^{n}=N_{2}^{n}=1 / 1500$ for this interval.

\section{CONCLUSIONS}

We have proved that issues of Elko spinor fields in 5D can be solved in $6 \mathrm{D}$ brane-worlds. We analyse the Elko fields in a thin and a thick string-like scenarios in $6 \mathrm{D}$, where one uses two type of 


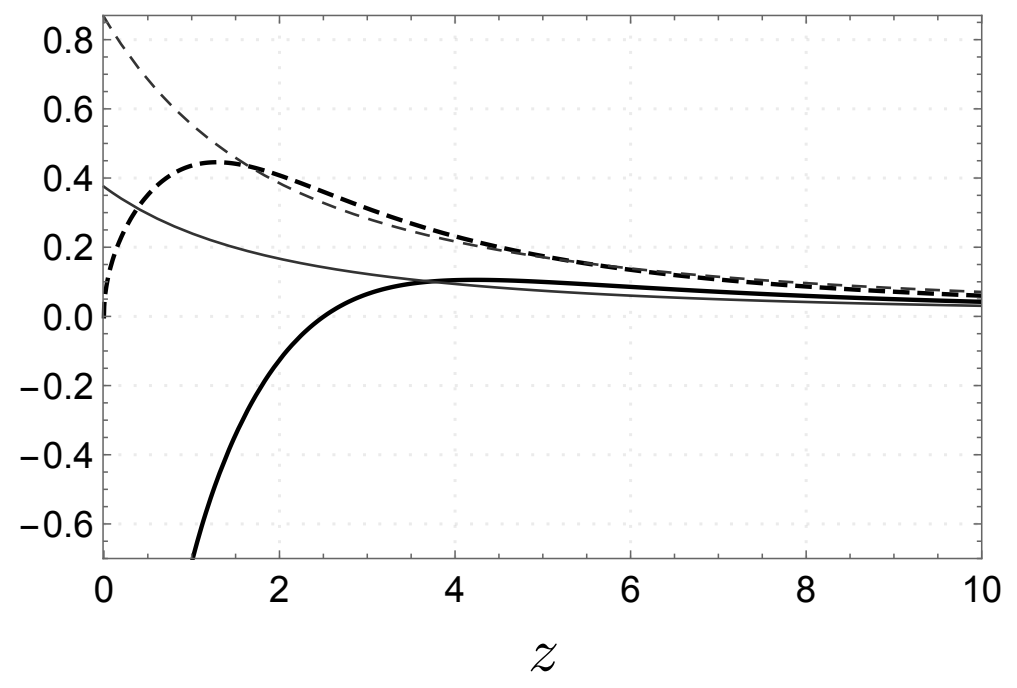

Figure 6: Plots for potentials $U(z)$ (full lines) for HC thick model (black line) and for GS thin model (gray line), and normalised massless modes $\varepsilon_{0}(z)$ (dashed lines) for HC thick model (dashed black line) and for GS thin model (dashed gray line), all cases for $c=0.5$.

scalar fields to confine the zero mode, turning the massive modes real-valued. The results in the GS thin model have been analytically achieved, whereas in the HC model the results were only numerically valued. Finally, we generalised the result for all string-like metrics in 6D. For future works, we propose the generation of a $6 \mathrm{D}$ model by a topological abelian Higgs model, where the background exotic term can be naturally obtained from this geometry. The study of other types of coupling is also present among our aims. Moreover, further mass-dimension one fields (in 4D) or mass dimension 5/2 fields (in 6D), like the self-interacting ones for any spin [24] and singular spinor fields [54], are going to be analysed in a 6D model context, accordingly. It is also worth to point out that given that Elko is a prime dark matter candidate, the associated KK modes should be localized to the brane a posteriori. However, it might live a priori in the bulk, since we have shown that the zero mode can be trapped on the brane. It would explain why dark matter only weakly interacts with matter.

\section{Acknowledgments}

CASA thanks CNPq for financial support through grants 305766/2012-0 and 448142/2014-7. DMD thanks to Projeto CNPq UFC-UFABC 304721/2014-0 and CAPES. RdR is grateful to CNPq 
grant No. 303293/2015-2, and to FAPESP Grant No. 2015/10270-0.

[1] Randall L. Sundrum R. Phys. Rev. Lett. 83, 3370 (1999).

[2] Randall L. Sundrum R. Phys. Rev. Lett. 83, 4690 (1999).

[3] Cartas-Fuentevilla R., Escalante A., Germán G., Herrera-Aguilar A. Mora-Luna R. R. JCAP. 1605, no. 05, 026 (2016).

[4] Cruz W. T., Maluf R. V., Dantas D. M. Almeida C. A. S. Annals Phys. 37549 (2016).

[5] Ikeda T. N., Lucas A., Nakai Y. JHEP 1604007 (2016).

[6] Casadio R. da Rocha R. Phys. Lett. B 763434 (2016).

[7] Correa R. A. C., Dantas D. M., Almeida C. A. S. da Rocha R. Phys. Lett. B 755358 (2015).

[8] Correa R. A. C., Dantas D. M., Moraes P. H. R. S, A. de Souza Dutra. Almeida C. A. S. arXiv:1607.01710 [hep-th].

[9] Megias E., Panico G., Pujolas O. Quiros M. JHEP 1609118 (2016).

[10] Chen P., Ding G. J., Rojas A. D., Vaquera-Araujo C. A. Valle J. W. F. JHEP 1601007 (2016).

[11] Gherghetta T. Shaposhnikov M. E. Phys. Rev. Lett. 85240 (2000).

[12] Silva J. E. G., Santos V. Almeida C. A. S. Class. Quant. Grav. 30025005 (2013).

[13] Veras D. F. S., Silva J. E. G., Cruz W. T. Almeida C. A. S. Phys. Rev. D 91065031 (2015).

[14] Dantas D. M., Veras D. F. S., Silva J. E. G. Almeida C. A. S. Phys. Rev. D 92104007 (2015).

[15] Araujo J. C. B., Veras D. F. S., Dantas D. M. Almeida C. A. S. arXiv:1610.08124 [hep-th].

[16] Oda I. Phys. Lett. B 496113 (2000).

[17] Costa F. W. V., Silva J. E. G. Almeida C. A. S. Phys. Rev. D 87125010 (2013).

[18] Torrealba R. S. Phys. Rev. D 82024034 (2010).

[19] Santos V., Almeida C. A. S. Phys. Lett. B 7181114 (2013).

[20] Rizzo T. G. JHEP 1011156 (2010).

[21] Liu Y. -X., Zhao L., Duan Y. -S. JHEP 0704097 (2007).

[22] Gogberashvili M., Midodashvili P. Singleton D. JHEP 0708033 (2007).

[23] Frère J. M., Libanov M., Mollet S. Troitsky S. JHEP 1308078 (2013).

[24] Lee C. Y. Dias M. Phys. Rev. D 94065020 (2016).

[25] Liu Y. X., Zhou X. N., Yang K. Chen F. W. Phys. Rev. D 86064012 (2012).

[26] Dias M., de Campos F. Hoff da Silva J. M. Phys. Lett. B 706352 (2012).

[27] Ahluwalia D. V., Lee C. Y. Schritt D. Phys. Rev. D 83065017 (2011).

[28] Fabbri L., Phys. Lett. B 704255 (2011).

[29] Ahluwalia D. V. and Nayak A. C. Int. J. Mod. Phys. D 231430026 (2015).

[30] Ahluwalia D. V. and Horvath S. P. JHEP 1011078 (2010).

[31] Fabbri L. Gen. Rel. Grav. 431607 (2011). 
[32] Ahluwalia D. V., Lee C. Y. and Schritt D. Phys. Rev. D 83065017 (2011).

[33] Ahluwalia D. V., Lee C. Y. and Schritt D. Phys. Lett. B 687248 (2010).

[34] Boehmer C. G., Annalen Phys. 1638 (2007).

[35] Agarwal B., Jain P., Mitra S., Nayak A. C. Verma R. K. Phys. Rev. D 92075027 (2015).

[36] da Rocha R., Hoff da Silva J. M. EPL 10750001 (2014).

[37] Cohen A. G., Glashow S. L. Phys. Rev. Lett. 97021601 (2016).

[38] Hoff da Silva J. M., S. H. Pereira JCAP 03009 (2014).

[39] Fabbri L. Phys. Lett. B 704255 (2011).

[40] Jardim I. C., Alencar G., Landim R. R. Costa Filho R. N. Phys. Rev. D 91085008 (2015).

[41] Cruz W. T., Gomes A. R. Almeida C. A. S. Eur. Phys. J. C 711790 (2011).

[42] Dantas D. M., Silva J. E. G. Almeida C. A. S. Phys. Lett. B 725425 (2013).

[43] Sousa L. J. S., Silva C. A. S., Dantas D. M. Almeida C. A. S. Phys. Lett. B 73164 (2014).

[44] Bernardini A. E., da Rocha R. Phys. Lett. B 717238 (2012).

[45] da Rocha R., Bernardini A. E., Hoff da Silva J. M. JHEP 04110 (2011).

[46] Avis S. J., Isham C. J. Nucl. Phys. B 156441 (1979).

[47] Petry H. R. J. Math. Phys. 20231 (1979).

[48] Avis S. J. Isham C. J. Comm. Math. Phys. 72103 (1980).

[49] Hawking S. W. Nucl. Phys. B 144349 (1978).

[50] Christensen S. M. Duff M. J. Nucl. Phys. B 14611 (1978).

[51] Banach R. J. Phys. A 14901 (1981).

[52] Ford L. H. Phys. Rev. D 21933 (1980).

[53] M. Giovannini, H. Meyer M. E. Shaposhnikov, Nucl. Phys. B 619615 (2001).

[54] Coronado Villalobos C. H., Hoff da Silva J. M. da Rocha R. Eur. Phys. J. C 75266 (2015). 